ESJ Social Sciences

\title{
El enojo desde la perspectiva de un grupo de niños y niñas mexicanos
}

Norma Angélica Ortega-Andrade, Dra.

Linda María Viramontes-Martínez, Lic.

David Jiménez-Rodríguez, Dr.

Universidad Autónoma del Estado de Hidalgo, México

Doi:10.19044/esj.2021.v17n5p51

Submitted: 05 December 2020

Accepted: 18 January 2021

Published: 28 February 2021
Copyright 2021 Author(s)

Under Creative Commons BY-NC-ND

4.0 OPEN ACCESS

Cite As:

Ortega-Andrade N.A., Viramontes-Martínez L.M. \& Jiménez-Rodríguez D. (2021). El enojo desde la perspectiva de un grupo de niños y niñas mexicanos. European Scientific Journal, ESJ, 17(5), 51. https://doi.org/10.19044/esj.2021.v17n5p51

\section{Resumen}

Este estudio tuvo como objetivo explorar las diferentes vivencias que los niños experimentan en torno a la emoción del enojo y analizar los factores que lo predisponen, así como las acciones que lleva a cabo para regular esta emoción. El estudio es cualitativo bajo un enfoque fenomenológico. Participaron un total de 34 niños y niñas de 9 a 12 años de los cuales 16 (47\%) de ellos fueron niños y 18 (53\%) fueron niñas. El muestreo fue noprobabilístico por conveniencia. La técnica para recolectar los datos fue un cuestionario conformado por nueve preguntas, que refiere a las cogniciones, conductas y sentimientos con respecto al enojo, el cual se aplicó en pequeños grupos conformados por 4 niños y/o niñas. Los resultados muestran que desde la perspectiva de los niños el enojo es sentirse irritado, expresarlo con gestos o respuestas agresivas, y la forma de regular su enojo es tratando de tranquilizarse o bien buscar una solución. Se concluye que las respuestas de los niños son más reactivas que anticipatorias.

Palabras clave: Emoción de enojo, niños, conceptualización, regulación emocional 


\title{
Anger from the Perspective of a Group of Mexican Boys and Girls
}

Norma Angélica Ortega-Andrade, Dra.

Linda María Viramontes-Martínez, Lic.

David Jiménez-Rodríguez, Dr.

Universidad Autónoma del Estado de Hidalgo, México

\begin{abstract}
This study aimed to explore the different experiences that children experience around the emotion of anger and to analyze the factors that predispose it, as well as the actions carried out to regulate this emotion. The study is qualitative under a phenomenological approach. A total of 34 boys and girls between the ages of 9 and 12 participated, of which $16(47 \%)$ of them were boys and $18(53 \%)$ were girls. The sampling was non-probabilistic for convenience. The technique to collect the data was a questionnaire made up of nine questions, which refers to cognitions, behaviors and feelings regarding anger, which was applied in small groups made up of 4 boys and / or girls. The results show that, from the children's perspective, anger is feeling irritated, expressing it with gestures or aggressive responses, and the way to regulate their anger is by trying to calm down or find a solution. It is concluded that the children's responses are more reactive than anticipatory.
\end{abstract}

Keywords: Anger emotion, children, conceptualization, emotional regulatio

\section{Introduction}

Las emociones como parte fundamental del ser humano son estados afectivos que permiten experimentar diversas reacciones que pueden ser de tipo psicológico, fisiológico o conductual. Éstas desempeñan un papel clave en el comportamiento del ser humano, en su aprendizaje y en su interacción social; su función principal es la adaptación a determinados estímulos del entorno como clave para la supervivencia (Alcazar-Olán et al., 2015; Fernández-Abascal \& Jiménez, 2010; Ramírez-Luna, 2018).

Así entonces, la capacidad para identificar y discriminar las emociones desde la niñez es fundamental para aprender a comprender y regular sus emociones. Un déficit en esta capacidad de acuerdo con Batty y Taylor (2006) puede derivar en problemas de adaptación social y convertirse en un factor de riesgo para el desarrollo de posibles psicopatologías en la edad adulta. Sin embargo, la manera de responder a cada una de éstas dependerá en gran medida del entorno sociocultural y las herramientas que este le brinde, así como del propio temperamento del niño. Jiménez-Rodríguez y Osorio (2016) consideran 
que una de las emociones más complejas y tal vez la de mayor dificultad en torno a su manejo y regulación adecuada es la emoción del enojo.

El enojo de acuerdo con Álvarez (2010), se puede entender como una fuerza interna que surge como respuesta ante diversas circunstancias y condiciones que ocasionan tensión, producen malestar o frustración. Al igual que las demás emociones, el enojo representa una manera natural de reaccionar ante una determinada situación que le alerta de una amenaza, como puede ser la presencia de alguna injusticia (Cupul, 2018; Dahab et al., 2007). Así mismo, el enojo según Spielberg (1999) es un estado emocional que varía en intensidad, el cual pude ir desde una irritación leve hasta una furia e ira intensa. Al igual que todas las emociones ésta se acompaña de cambios psicológicos y biológicos que se manifiestan en la conducta humana.

En el caso específico de los niños el enojo se expresa de distintas maneras, pueden sentirse mal o representarlo tanto a nivel físico como psicológico. Es fácil reconocer cuando se encuentran en este estado, pues suelen ser hostiles con los demás. En los niños de edad escolar específicamente, las conductas agresivas que suelen manifestar comúnmente son los insultos, las burlas, ridiculizar a otros y otro tipo de acciones que incluyen violencia física. En este sentido, este comportamiento puede llegar a ocasionarles problemas en su entorno familiar, con sus compañeros y amigos, así como dificultades en su rendimiento escolar (Steinberg, 2018).

De igual forma, la expresión y manifestación del enojo en algunos niños se expresa a través del llanto, el mal humor, las expresiones faciales y muecas, así como evitando o evadiendo a la persona que los provoca, o bien, algunos buscan el apoyo de un adulto como refugio o consuelo (Marion, 1997). Fabes y Eisenberg (1992) consideran que las principales situaciones de enojo en los niños tienen que ver con la invasión de su espacio personal, la agresión física, los conflictos verbales y el rechazo que se puede suscitar, por ejemplo, en el aula escolar.

Es importante mencionar que cuando el enojo es leve éste cumple con las funciones positivas de alerta y protección; de manifestar una insatisfacción, motivar a una acción correctiva y mostrar comportamientos adaptativos para enfrentar las situaciones adversas; lo cual muestra que cuando el enojo es manejado de forma equilibrada éste favorece la adaptación al medio (Cupul, 2018). A esta característica del enojo positivo Alcázar-Olán (2017) la denomina enojo sano. Este autor menciona que este tipo de enojo es "útil para detectar injusticias, resolver problemas y cumplir metas positivas a largo plazo, el enojo sano tiene una intensidad controlable que se activa positivamente para encontrar soluciones de forma constructiva" (p.68); esto es, será saludable siempre y cuando dicha emoción se utilice para detectar injusticias, marcar límites o reclamar los derechos humanos (Cupul, 2018; Dahab et al., 2007). 
En cambio, cuando la intolerancia es mal manejada en la niñez y se hace presente el enojo por no conseguir sus deseos de manera inmediata, ésta genera inseguridad o baja autoestima, provocando la sensación de malestar corporal general y de enojo consigo mismo (Esteban, 2018).

Así entonces, la capacidad para identificar y comprender esta emoción en la niñez es el referente a partir del cual aprenderá a regular esta emoción, lo cual es muy conveniente ya que esto evitará respuestas violentas, siendo menos probable que también sean víctimas de violencia. Cuando los niños aprenden formas constructivas de solucionar los problemas, enfrentar los desacuerdos y controlar su enojo se está actuando en favor de la prevención de la violencia (American Psychological Association). De ahí la importancia de explorar desde la propia vivencia de los niños y no de los adultos, la forma en qué ellos perciben el enojo en sí mismos, cómo lo reconocen, cómo reaccionan cuando están enojados y qué hacen para regularlo.

Los estudios en torno al enojo en la niñez, se han abocado a la implementación de programas de intervención cognitivo conductual (Mytton et al. 2002; López 2014; Pérez, et al., 2008), así como a la identificación, comprensión y expresión del mismo a través de la literatura infantil, con el uso de los cuentos (Gómez, 2020; Moreno, 2017; Pérez 2017, Vicario, 2019), o el diseño de instrumentos para medir la ira en niños mexicanos (Alcázar et al.,2012; Cupul, 2018), pero poco se ha abordado el enojo desde la propia mirada del niño. De hecho, como refiere Martínez (2016), son muy pocos los estudios que se han realizado en torno a las estrategias de regulación emocional que utilizan los niños escolarizados. Al respecto solo se encontró un estudio realizado por Oolup et al. en 2016 quienes exploraron el enojo desde las propias experiencias en niños y niñas de 8 y 9 años a través de entrevistas semiestructuras en grupos focales. Sin embargo en México, no existe un estudio que permita reconocer cómo los niños conciben el enojo, lo cual es de observar dado los altos índices de violencia que se viven en el país. Tampoco hay estudios que permitan observar el proceso que siguen los niños para regular esta emoción, por lo que se consideró el modelo procesual de regulación emocional de Gross y Thompson (2007) para ubicar de acuerdo con este modelo en qué etapa del proceso se ubicaban las respuesta de los niños.

Como refiere la American Psychological Association, además de Ersan (2020) un enojo mal manejado y mal regulado puede causar comportamientos frecuentemente agresivos en etapas posteriores, pero también como señala Vicario (2019) es en edades tempranas cuando se puede enseñar a los niños a lidiar de forma positiva con el enojo como parte de su desarrollo integral.

Tomando en cuenta lo importante que es la identificación de esta emoción en edades tempranas y de la niñez, el presente estudio tuvo como 
objetivo explorar las diversas experiencias derivadas del enojo, su concepción y forma de regularlo en los niños y niñas de 9 a 12 años, a fin de contar con información que contribuya al estudio de esta emoción en este grupo etario y poder generar estrategias de intervención en regulación emocional para los niños y niñas mexicanos(as). Además de tener las consideraciones para el momento de confinamiento por el COVID-19, en los núcleos familiares donde de acuerdo con estadísticas del Instituto Nacional de Estadística y Geografía (INEGI), revelan estas condiciones de enojo que detonan conflictos al seno de la familia, en particular con los niños de las edades de este estudio.

\section{Método}

Participantes

En el estudio participaron 34 niños y niñas de entre 9 a 12 años de edad de una escuela primaria pública de la ciudad de Pachuca, Hidalgo, México. Del total de participantes, 5 (15\%) fueron de 4to grado; 17 (50\%) fueron de 5to grado y 12 (35\%) fueron de 6to grado; por género $16(47 \%)$ de ellos fueron niños y 18 (53\%) fueron niñas. El muestreo fue no-probabilístico por conveniencia. Como parte de los apectos éticos de la investigación se consideró la autorización firmada (consentimiento informado) de los padres o tutores legales; así como el asentimiento informado del propio alumno.

Diseño de la investigación

Con una metodología cualitativa y un enfoque fenomenológico (Hernández \& Mendoza, 2018), se indagó en las concepciones y formas de regular el enojo en los niños y niñas de este rango de edad. El enfoque fenomenológico se caracteriza por explorar, describir y comprender lo que los individuos tienen en común de acuerdo con sus experiencias con un determinado fenómeno.

Técnica de recolección de datos

Entrevista grupal en formato estructurado, el cual fue elaborado por Ortega-Andrade (2018), quien es una de las autoras de este artículo. Los planteamientos surgieron de los recursos regulatorios individuales propuestos por Sánchez-Aragón (2010) en su modelo de regulación emocional: percepción, expresión y entendimiento emocional, que fueron retomados por esta autora de Salovey y Mayer (1990), y que en su modelo los considera como capacidades precursoras para poder ejercer la regulación. De igual forma, el planteamiento de dichas preguntas se sustenta en el proceso de la regulación emocional propuestos por Gross y Thompson (2007).

La entrevista consta de 9 preguntas tipificadas en conocimientos, percepciones, condiciones y expresión de sentimientos, planteadas en los siguientes cuestionamientos: (1) “¿Sabes qué es el enojo?”, cuya finalidad fue 
conocer el concepto y entendimiento de la emoción desde la perspectiva del niño; (2) “¿Qué te hace sentir enojado?”, para identificar los factores que lo promueven; (3) “¿Cómo sabes que estás enojado?”, para conocer la percepción de la emoción; (4) “¿En qué lugar te sientes enojado?”, conocer en dónde se presenta el enojo; (5) “HHay alguien que te haga sentir enojado?", para indagar acerca de las personas de su entorno y el significado que éstas tienen para él; (6) “¿Ha pasado algo que te ponga muy enojado?”, para conocer la situación o acontecimientos detonantes; (7) “¿Qué haces cuando estás muy enojado?", para conocer la conducta o bien, las herramientas con las que cuenta para su gestión; (8) “¿Cómo demuestras que estás enojado?”, para conocer la expresión y manifestación de la emoción; (9) “¿Qué piensas cuando estás enojado?", para identificar el significado que le da a la expresión del enojo. La información de las entrevistas se recabo mediante audiograbaciones. Se consideró esta técnica porque al entrevistar a los niños, es posible que de forma espontánea expresen lo que piensan, les hace enojar y qué hacen para regular su enojo.

\section{Procedimiento}

Toda vez aceptada la propuesta del proyecto de investigación por parte de las autoridades de la escuela primaria y obtenido el consentimiento informado por parte de los padres de familia, se llevaron a cabo las entrevistas con los alumnos de los grados seleccionados de la siguiente manera:

1. Se entregó a la directora del plantel educativo la lista con los nombres de los niños y niñas que fueron autorizados por sus padres para participar en el estudio. Con esa lista, una profesora asignada por la directora, fue ubicando a los niños y niñas quienes fueron llevados en grupos de 3 al salón que fue asignado para realizar las entrevistas. Los grupos se fueron integrando con base en la disposición que existía para que los niños y niñas pudieran salir un momento de su salón de clases, por lo que los grupos quedaron conformados, en ocasiones, por niños y niñas de diferentes grupos escolares.

2. Esta misma forma de integrar los grupos se llevó a cabo hasta que se agotó a todos los partipantes de la lista en el transcurso cinco días consecutivos, durante un lapso de cuatro horas por día, que permanecía la psicóloga en el plantel. Las entrevistas fueron grabadas, con una duración aproximada de 30-40 minutos, en una única sesión por grupo.

3. Cuando los niños y/o niñas ya se econtraban instaldos en el aula que fue asignada para poder llevar a cabo las entrevistas, se daba inicio con la sesión. Ésta comenzaba con la presentación de la psicóloga quien realizó las entrevistas e informó a los niños y niñas el objetivo de su participación en el estudio. Una vez que ellos aceptaron su colaboración, se plantearon las preguntas una por una danto tiempo a 
que cada participante diera su respuesta. Cuando se agotaban las preguntas, la psicóloga agradecía su participación, preguntando si querían agregrar algo más. Posteriormente los niños eran trasladados a sus aulas por la profesora que estaba de apoyo.

4. Finalizada la grabación de todas las entrevistas el siguiente paso fue transcribirlas; posteriormente la información fue categorizándose de manera sistemática, para su análisis considerando los siguientes ejes temáticos (que corresponden a cada una de las preguntas planteadas en la entrevista):

- Conceptualización y entendimiento de la emoción del enojo.

- Autopercepción de la emoción de enojo

- Factores que lo promueven

- Situación o acontecimiento detonante de enojo

- Conexto de enojo

- Personas que detonan enojo

- Gestión del enojo

- Expresión y manifestación del enojo

- Signicado a la expresión del enojo

Análisis de los datos

El análisis de la información se realizó de acuerdo con el enfoque fenomenológico empírico, el cual según Creswell et al. (2007), se centra en describir las experiencias de los participantes.

Una vez que se obtuvo la información, se transcibió textualmente cada una de las respuestas dadas por los niños y las niñas, se porcedió a llevar a cabo el análisis. Se remarco de forma artesanal con diferentes colores acorde al código, los enunciados más importantes para comprender cómo los participantes concebían, percibían, comprendían y expresaban y gestionaban el enojo. Se agruparon los enunciados por su frecuencia. Se describió las experiencias de acuerdo con los enunciados y se generaron las categorías. La información se presenta en tablas según cada pregunta que corresponden a los ejes temáticos.

Posteriormente con base en las experiencias detectadas sobre ¿Qué hacen cuando están enojados? y ¿qué piensan cuando están enojados?

se ubicó con el modelo procesual de regulación emocional de Gross y Thompson (2007), en cuál de los procesos se encuentran las acciones que llevan a cabo para regular el enojo.

Este modelo sigiere que existen dos mecanismos básicos en el proceso de generación de una emoción: las estrategias focalizadas en el antecedente (son aquellas se que se ponen en marcha antes de que las tendencias de respuesta emocional estén completamente activadas), y estrategias focalizadas 
en la respuesta o consecuente (se refiere a las estrategias que se implementan una vez que las respuestas emocionales han sido generadas).

Como parte de las estrategias antecedentes, se encuentra:

- La selección de la situación. Implican la realización de ciertas acciones que permiten a la persona ser o no parte de una situación y las respuestas emocionales que éstas desencadenarían.

Respecto de las estrategias enfocadas en la respuesta:

- Modificación de la situación. Se utiliza cuando se quiere cambiar el impacto emocional de una situación y para lograrlo se recurre a determinadas acciones específicas. Martínez (2016), expone que un ejemplo de este tipo de estrategia es cuando los niños en medio de una situación de discusión con sus padres, se ponen tristes o comienzan a llorar para poder así bajar el tono emocional de la interacción.

- Despliegue de la atención. Se focaliza la atención en algún aspecto específico de la situación que puede influir sobre las emociones. Se encuentran dentro de ésta, la distracción y la concentración (que se refiere a la atención focalizada en ciertas características emocionales de la situación, como puede ser la rumiación).

- Cambio cognitivo. Es cuando se modifica la evaluación realizada sobre una situación determinada, con el objetivo de variar su significado emocional, cambiando lo que se piensa sobre dicha situación o sobre la propia capacidad para manejar las demandas de ésta.

- Modulación de la respuesta. Respuestas fisiológicas, esperenciales o comportamentales ante una respuesta emocional que se ha iniciado. Una forma de modulación de la respuesta, es la supresión de la expresión emocional, que es la inhibición de la expresión comportamental de una emoción, lo cual no significa que haya una disminución de la experiencia emocional negativa.

Resultados

Se obtuvieron una serie de respuestas concernientes a cada una de las 9 preguntas realizadas a los 34 niños y niñas. Se sistematizaron y categorizaron de la manera que se muestra a continuación.

¿Sabes qué es el enojo? Esta pregunta fue realizada como ya se dijo previamente, con la finalidad de conocer a fondo la conceptualización y entendimiento de la emoción desde la perspectiva del niño. Cabe resaltar que los niños de 4to año ofrecieron respuestas sencillas a esta pregunta, mientras que los niños mayores utilizaron conceptos más elaborados como "frustración", "estrés" e "irritabilidad", lo que resulta comprensible debido a la propia madurez del niño, ya que a mayor edad mayor complejidad y 
abstracción en su lenguaje. Del total de 34 alumnos en esta pregunta se abstuvieron de responder tres niños (ver Tabla 1).

Tabla 1. ¿Sabes qué es el enojo?

\begin{tabular}{lcc}
\hline Respuestas & Frecuencia & \% \\
\hline Irritabilidad & 9 & 29.0 \\
Sentirse mal & 7 & 22.6 \\
No querer hablar con nadie & 7 & 22.6 \\
Cuando te enojas & 3 & 9.7 \\
Frustrarse & 2 & 6.5 \\
No sé & 2 & 6.5 \\
Sentirse estresado & 1 & 3.1 \\
\hline Total & 31 & 100
\end{tabular}

¿Qué te hace sentir enojado? Se observaron en su mayoría los insultos, el mal trato ejercido por los demás (su entorno), así como la invasión de su espacio como factores relevantes. Del total de 34 alumnos se abstuvieron de responder cuatro niños (ver Tabla 2).

Tabla 2. ¿Qué te hace sentir enojado?

\begin{tabular}{lcc}
\hline Respuesta & Frecuencia & \% \\
\hline El maltrato de los demás & 11 & 36.7 \\
Insultos & 7 & 23.3 \\
Cuando toman cosas sin mi permiso & 4 & 13.3 \\
Regaño & 3 & 10.0 \\
No lograr cosas / metas & 2 & 6.7 \\
Que le falten el respeto a mi familia & 2 & 6.7 \\
Cuando me hacen bromas & 1 & 3.3 \\
\hline Total & 30 & 100 \\
\hline
\end{tabular}

* Respondieron 30 de 34 participantes

¿Cómo sabes que estás enojado? La mayoría de los niños reconoció el saberse enojados por medio de las reacciones fisiológicas, sin embargo cabe resaltar que en esta pregunta existió poca cantidad de respuestas. Del total 34 alumnos, se abstuvieron de responder trece niños (ver Tabla 3 ).

Tabla 3. ¿Cómo sabes que estás enojado?

\begin{tabular}{lcc}
\hline Respuesta & Frecuencia & \% \\
\hline Sensación de calor en la piel y el estómago & 6 & 28.6 \\
Por mi expresión facial o mis gestos & 6 & 28.6 \\
Cuando soy grosero & 4 & 19.0 \\
Porque las personas me lo dicen & 2 & 9.5 \\
Porque no me siento feliz & 2 & 9.5 \\
Porque tengo ganas de llorar o gritar & 1 & 4.8 \\
\hline Total & 21 & 100 \\
\hline
\end{tabular}

* Respondieron 21 de 34 participantes 
¿En qué lugar te sientes enojado? Es importante destacar que la mayoría refirió tanto "la casa" como "su escuela" como los lugares donde mayormente se sienten enojados. Del total 34 alumnos se abstuvieron de responder cuatro niños (ver Tabla 4).

Tabla 4. ¿En qué lugar te sientes enojado?

\begin{tabular}{lcc}
\hline Respuesta & Frecuencia & \% \\
\hline Mi casa & 12 & 40.0 \\
Escuela & 9 & 30.0 \\
Ninguno & 4 & 13.4 \\
Cuando me molestan & 3 & 10.0 \\
Hospital & 1 & 3.3 \\
Donde hay basura & 1 & 3.3 \\
\hline Total & 30 & 100 \\
\hline
\end{tabular}

* Respondieron 30 de 34 participantes

¿Hay alguien que te haga sentir enojado? Los resultados muestran que en la mayoría de los casos el "hermano/a" del niño es visto como la persona que genera el enojo. Del total 34 alumnos se abstuvieron de responder nueve niños (ver Tabla 5).

Tabla 5. ¿Hay alguien que te haga sentir enojado?

\begin{tabular}{lcc}
\hline Respuesta & Frecuencia & \% \\
\hline Hermano / a & 11 & 44.0 \\
Nadie & 6 & 24.0 \\
Compañero de la escuela & 5 & 20.0 \\
Padres & 2 & 8.0 \\
Primo & 1 & 4.0 \\
\hline Total & 25 & 100 \\
\hline \multicolumn{3}{c}{}
\end{tabular}

¿Ha pasado algo que te ponga muy enojado? Del total 34 alumnos se abstuvieron de responder ocho niños (ver Tabla 6).

Tabla 6. ¿Ha pasado algo que te ponga muy enojado?

\begin{tabular}{lcc}
\hline Respuesta & Frecuencia & \% \\
\hline Nada & 11 & 42.3 \\
Que no respeten mis cosas & 4 & 15.4 \\
No lograr objetivos / metas & 3 & 11.6 \\
Regaño & 2 & 7.7 \\
Que hablen de mi físico & 2 & 7.7 \\
Que le falten el respeto a mi familia & 2 & 7.7 \\
Ser ignorado & 1 & 3.8 \\
Mi compañero se sentó en mi lugar & 1 & 3.8 \\
\hline Total & 26 & \\
& & 100 \\
\hline
\end{tabular}

* Respondieron 26 de 34 participantes 
¿Qué haces cuando estás muy enojado? Esta pregunta fue realizada con el fin de identificar la conducta o acción llevada a cabo, así como las herramientas que el niño utiliza para su manejo y gestión. Aquí las respuestas fueron sumamente variadas. Como se pudo observar, la gran mayoría de los niños no contaban con las herramientas o formas apropiadas para el manejo y regulación del enojo, por el contrario, sus acciones revelaron una inadecuada gestión del mismo. Del total 34 alumnos se abstuvieron de responder tres niños (ver Tabla 7).

Tabla 7. ¿Qué haces cuando estás muy enojado?

\begin{tabular}{lcc}
\hline Respuesta & Frecuencia & \% \\
\hline Encerrarme en mi cuarto & 7 & 22.7 \\
Aventar / quebrar cosas & 5 & 16.1 \\
Tratar de tranquilizarme & 5 & 16.1 \\
Llorar & 4 & 12.9 \\
Pelear con alguien & 4 & 12.9 \\
Acostarse / dormir & 4 & 12.9 \\
Golpear & 1 & 3.2 \\
Decirle a su papá & 1 & 3.2 \\
\hline Total & 31 & 100 \\
\hline
\end{tabular}

* Respondieron 31 de 34 participantes

¿Cómo demuestras que estás enojado? Esta pregunta se hizo con la intención de conocer la expresión y la forma de manifestación en los niños. Los datos resultantes corroboran lo expuesto con anterioridad con respecto a la falta de habilidades o medios viables con los que los niños de esta población cuentan. Del total de 34 alumnos se abstuvo de responder un solo niño (ver Tabla 8).

Tabla 8. ¿Cómo demuestras que estás enojado?

\begin{tabular}{lcc}
\hline Respuesta & Frecuencia & \% \\
\hline Por mi expresión facial / por mis gestos & 10 & 30.3 \\
Rompiendo cosas & 5 & 15.2 \\
Gritando & 5 & 15.2 \\
Golpeando & 4 & 12.1 \\
Ignorando si me hablan & 4 & 12.1 \\
Llorando & 3 & 9.0 \\
Por mi carácter & 2 & 6.1 \\
\hline Total & 33 & 100 \\
\hline
\end{tabular}

* Respondieron 33 de 34 participantes

¿Qué piensas cuando estás enojado? De 34 niños encuestados todos en su totalidad respondieron a esta pregunta (ver tabla 9). 
Tabla 9. ¿Qué piensas cuando estás enojado?

\begin{tabular}{lcc}
\hline Respuesta & Frecuencia & \% \\
\hline Que les pase algo malo a los que provocaron eso & 9 & 26.6 \\
Qué hacer ante la situación / buscar una solución & 5 & 14.7 \\
En porqué me hicieron enojar & 5 & 14.7 \\
Nada & 4 & 11.8 \\
Que debo tranquilizarme & 3 & 8.8 \\
Matar & 2 & 5.9 \\
Golpear & 2 & 5.9 \\
No querer vivir & 1 & 2.9 \\
Hermano / hermana & 1 & 2.9 \\
No estar cerca de quien me provocó el enojo & 1 & 2.9 \\
Jugar & 1 & 2.9 \\
\hline Total & 34 & 100 \\
\hline
\end{tabular}

* Respondieron los 34 participantes

Con respecto al análisis de las respuestas de los niños y niñas con el modelo de regulación emocional de Gross y Thompson (2007), éstas se pueden ubicar en estrategias enfocadas en la respuesta, es decir, una vez que se ha presentado la situación causante de enojo.

Si se analiza específicamente qué hacen cuándo están enojados, de acuerdo con este modelo, sus respuestas se ubican en estrategias de modificación de la situación, cuando refieren retirarse del lugar fìsico, como lo es el encerrarse en su cuarto, o bien desplegar la atención al acostarse o dormir. Sin embargo, también hay quienes se enfocan en la modulación de la respuesta, como lo es el tratar de tranquilizarse. No obstante, es de obsevar que existen respuestas reactivas de una expresión del enojo con alta intensidad como aventar o quebrar cosas, golpear o pelear con alguien.

De igual forma, se puede indetificar que algunas de sus respuestas referentes a qué piensan cuando están enojados, se ubican en el proceso de desplazamiento de la atención al centrar sus pensamientos en la situación que ocasionó el enojo. También es evidente, aunque en el menor de los casos, hay quienes podrían encontrarse en un paso para el proceso de cambio cognitivo, al buscar una solución ante la situación, o bien quienes piensan que deben tranquilizarse, como una forma de modular su respuesta.

\section{Discusión}

Los resultados del presente estudio, dan cuenta de que los niños y las niñas que participaron en la investigación, presentan tanto acciones regulatorias positivas como negativas ante el enojo, revelando incluso algunas conductas de riesgo, en tanto, son capaces de reconocer que el enojo les genera 
irritación y malestar, así como respuestas que se pueden considerar como agresivas (aventar o quebrar cosas, golpear o pelear con alguien).

Con respecto a las etapas del desarrollo emocional del niño y haciendo referencia al comportamiento o conductas presentadas en esta edad, González (2020) señala que esta etapa se caracteriza por un desajuste y variabilidad de las emociones, ya que los niños suelen experimentar sentimientos contradictorios como pueden ser amor-odio y soledad-compañía. Es en esta etapa en la cual empiezan a tener sentimientos que probablemente no habían experimentado antes, como el amor. Aquí, la amistad se vuelve primordial y se basa en la intimidad y confianza.

En este sentido, entre las respuestas expresadas en relación a los causas o condiciones que propician el enojo, se evidenció que los elementos principales lo conforman factores familiares, personales, como "no lograr metas o cosas" o bien cuando "toman cosas sin su permiso", "intimos" (de amistad), y con relación a su autoestima o seguridad, los insultos-regaños. Así pues, en lo concerniente a la pregunta acerca del lugar en el que sienten enojo, la gran mayoría de los niños manifestaron la casa y la escuela como los sitios donde más sentían enfado. Dicho lo anterior, Chorpita y Barlow (1998), señalan que el entorno familiar es el que contribuye a presentar en mayor cantidad elementos y factores que predisponen a la aparición y mantenimiento de la ira, ya que el núcleo familiar es el contexto más trascendental durante la infancia. De igual forma, como refieren Fabes y Eisenberg (1992) las principales situaciones de enojo en los niños tienen que ver con la invasión de su espacio personal, la agresión física, los conflictos verbales y el rechazo que se puede suscitar, por ejemplo, en el aula escolar.

En lo que respecta a la percepción del enojo, los niños tuvieron mayor dificultad al expresar el cómo saber que estaban enojados, pues existió una cantidad considerable de niños que no brindaron respuesta. Sin embargo, es importante añadir que la mayoría de los que si respondieron reconocieron saberse enojados a través de reacciones y respuestas fisiológicas. González (2020) asevera que en esta etapa los niños tienden a volverse más reservados con respecto a las emociones y la expresión de las mismas.

Asimismo puede inferirse que la carencia de respuestas pueda ser debido a que en esta edad, los niños aún tienen dificultad para expresar determinado tipo de conceptos con respecto a las "percepciones" y "sensaciones" derivadas de esta emoción, es decir, las sienten pero desconocen en algunos casos como expresarlas o referirse a ellas. No obstante, en otras circunstancias o contextos, los niños cuentan por el contrario con mayor fluidez y mayor abstracción en su lenguaje elaborando complejas respuestas, ya que su propio desarrollo cognitivo perteneciente a estos años (de 9 a 12 años), facilita que su pensamiento sea vuelva cada vez más crítico. Asimismo Piaget (2017), destaca en sus etapas del desarrollo cognitivo, que la etapa de 
las operaciones concretas, rango en el que se encuentra la población de niños encuestados, se caracteriza por el desarrollo en el razonamiento a través de la lógica, lo que lo lleva a distinguir entre realidad y ficción.

Por lo que se refiere a "quien" les genera enojo, nuevamente se reveló el impacto de la familia, así como el contexto social/escolar como determinantes, ya que la mayoría expresó que su hermano/a, así como un compañero de la escuela eran las personas que los hacían sentir enojo. Cotejando los hallazgos previos, Chorpita y Barlow (1998), refieren que la conducta agresiva se manifiesta y estabiliza a partir de la interacción y transacción continuas del niño con su entorno inmediato o núcleo, específicamente en las relaciones cotidianas como lo es su familia y sus compañeros. Es debido al propio desarrollo emocional de esta edad que los niños empiezan a tener la necesidad de desvincularse en el contexto afectivo de los padres, para crear su propia personalidad. Es entonces probable que se origine un entorno un tanto hostil en ocasiones dentro del ambiente familiar, ya que el niño buscará reafirmar su identidad y es debido a ello también impactará también en las relaciones con sus hermanos.

En relación con los acontecimientos o situaciones que les generan enojo, resulta interesante destacar que el mayor porcentaje de respuestas fue "nada", ya que previamente manifestaron diversos factores, contextos y elementos como causa principal. Se conjetura que posiblemente entendieron esta pregunta como similar. Empero, entre las respuestas restantes se encuentran nuevamente situaciones personales, escolares, y familiares tales como las expresadas con anterioridad.

En el aspecto perteneciente a las acciones llevadas a cabo ante esta emoción, se identifican diversas conductas, algunas de ellas asertivas y otras defavorables. Cabe resaltar que es de suma importancia indagar en este aspecto, pues advierte los recursos con los que cuenta el niño, así como la manera en la que este intenta manejar dicha emoción. En tanto que no se aprenda a regular tales explosiones agresivas, se originarán continuos conflictos en los distintos ámbitos sociales del niño (Steinberg, 2018).

Del mismo modo, en relación al tipo de manifestación o expresión del enojo, se logró corroborar o respaldar lo expuesto previamente, ya que las respuestas que se obtuvieron fueron similares. Los pensamientos generados a raíz del enojo también son diversos y muchos de ellos nos permiten una vez más confirmar la carencia de recursos que los niños conocen o tienen para aprender a manejar su enojo. Entre las respuestas están matar, no querer vivir, golpear y venganza. Por otro lado, destacan en particular tres respuestas por su contenido asertivo, "jugar", "que debo tranquilzarme" y "qué hacer ante la situación/buscar una solución", que podrían indicar una forma de regular el enojo, sin embargo, se identifican en menor medida características de un 
enojo sano, como refiere Alcázar-Olán (2017), sino más bien parecen respuestas reactivas ante las situaciones de enojo.

Estos resultados llevan a plantear la posibilidad de patrones aprendidos de los padres, quienes de acuerdo con Flores-Galaz y Sánchez-Aragón (2010), pueden llegar a responder ante el enojo de sus hijos de la siguiente manera: con restricción de la expresión, los regaños o bien, la muestra de indiferencia ante el enojo, además de manifestaciones positivas como el comunicar la causa del enojo, dar una solución, tranquilizarse o redireccionarlo, según un estudio realizado por estas autoras con 233 padres mexicanos. Sin embargo, se recomienda seguir investigar sobre el tema, pues de acuerdo con Ersan (2020), el enojo infantil mal manejado y mal regulado puede causar comportamientos frecuentemente agresivos en etapas posteriores y la prevención de la violencia es una condición social a la cual se necesita seguir atendiendo en México.

Respecto de las experiencias de enojo reportadas por los niños, ubicadas en el modelo de Gross y Thompson (2007), éstas son coincidentes con los comportamientos reportados por Sánchez-Aragón y Díaz-Loving (2009), en cuanto a la forma en que experimentan el enojo las personas jóvenes:

..cuando una persona siente desde una leve irritación hasta la furía o la ira, lo primero que hacen es alejarse del escenario donde puede surgir el estímulo de la experiencia de enojo (selección de la situación); posteriormente busca evadir la situación como una manera de cambiar el estado emocional (modificación de la situación), al no lograrlo trata de distraerse (despliegue de la atención) y para reafirmar esta postura, minimiza la importancia del evento al tratar de verla como algo más manejable (cambio cognoscitivo). En cuanto a la modulación de la respuesta, se puede observar entre otras respuestas agresivas (golpear cosas, ofender, apretar algo, aventar objetos y gritar) o bien, buscar calma y espacimiento. (pp. 267-268)

No obstante que las respuestas de los niños parecen ser similares a las reportadas por Sánchez-Aragón y Díaz-Loving (2009), se recomienda en futuros estudios analizar el proceso de regulación emocional que cada participante pueda tener a fin de corroborar si este mismo proceso se encuentra en los menores de este rango de edad, pues como se pudo apreciar, los participantes de este estudio, se enfocaron a las estrategias consecuentes y no al antecedente.

\section{Conclusión}

Los hallazgos encontrados en el presente estudio llevan a reflexionar en el papel que juegan los padres, los hermanos, así como el entorno escolar y social del niño, como un factor clave de soporte y guía para que éste aprenda a traducir y vivenciar el enojo como una función adaptativa a su medio, así 
como un recurso que le permita poder hacer frente a las diversas amenazas que se presenten.

Es importante mencionar que desde la perspectiva de los niños el enojo es sentirse irritado, expresarlo con gestos o respuestas agresivas, y la forma de regular su enojo es tratando de tranquilizarse o bien buscar una solución.

Los resultados de este estudio, solo son un referente para seguir investigando cualitativamente sobre el tema, de tal forma que la contextualización del enojo como emoción presente en la niñez, sea una condición para la intervención en la educación y en la regulación emocional.

\section{References:}

1. Alcázar-Olán, R. J. (2017). Enojarse: ¿cuándo es insano y cómo resolverlo? Revista Rúbricas, 11, 64-71. https://repositorio.iberopuebla.mx/bitstream/handle/20.500.11777/39 71/ENOJARSE.pdf?sequence=1

2. Alcázar-Olán, R. J., Deffenbacher, J. L., Hernández, L., \& Jurado, S. (2015). High and Low Trait Anger, Angry Thoughts, and the Recognition of Anger Problems. The Spanish Journal of Psychology, 18(84), 1-9. https://doi.org/10.1017/sjp.2015.84

3. Alcázar, R., Deffenbacher, J., Verónica Reyes, V., \& Pool, W. (2012, julio- diciembre). Desarrollo de un Inventario para la Medición de la Ira en niños mexicanos. Revista Colombiana de Psicología, 21, 3003313.

4. Álvarez, M. P. (2010). Enseñando a expresar la ira: ¿es una emoción positiva en la evolución de nuestros hijos? Pirámide.

5. American Psychological Association, A parenting program. https://www.apa.org/act/resources/espanol/enojo

6. Batty, M., \& Taylor M.J. (2006). The development of emotional face processing during childhood. Developmental. Science, 9 (2), 207-220.

7. Chorpita, B. F., \& Barlow, D. H. (1998). The development of anxiety: The role of control in the early environment. Psychological Bulletin, 124(1), 3-21. https://doi.org/10.1037/0033-2909.124.1.3.

8. Cupul, Ma. J. (2018). Validación de un inventario para la medición de la ira en niños meridanos (Tesis de maestría). Universidad Iberoamericana Puebla, México.

9. http://repositorio.iberopuebla.mx/licencia.pdf

10. Dahab, J., Minici, A., \& Rivadeneira, C. (2007). El enojo y su expresión. Revista de Terapia Cognitivo Conductual, 12, 1-5. http://cetecic.com.ar/revista/pdf/el-enojo-y-su-expresion.pdf.

11. Ersan, C. (2020). Physical aggression, relational aggression and anger in preschool children: The mediating role of emotion regulation. The 
Journal of General Psychology, 147:1, 18-42, https://doi.org/10.1080/00221309.2019.1609897

12. Esteban, E. (2018). Las emociones que los niños esconden tras la ira. Guía infantil. https://www.guiainfantil.com/educacion/conducta/8causas-de-enfado-en-los-ninos.

13. Fabes, R. A., \& Eisenberg, N. (1992). Young children's coping with interpersonal anger. Child Development, 63(1), 116-128. https://doi.org/10.1111/j.1467-8624.1992.tb03600.x.

14. Fernández-Abascal, E. G., \& Jiménez, M. P. (2010). Psicología de la emoción, Capítulo 1. En Férnandez-Abascal \& Jiménez (Eds.). Psicología de la Emoción (pp. 17-18). Universitaria Ramón Areces. https://www.cerasa.es/media/areces/files/book-attachment-2986.pdf.

15. Flores-Galaz, M.M., \& Sánchez-Aragón, R. (2010). Ontogenia y filogenia cultural de la regulación emocional. En R. Sánchez-Aragón (Ed.), Regulación emocional. Una travesía de la cultura al desarrollo de las relaciones personales ( $\mathrm{pp}$. 76-124). Universidad Nacional Autónoma de México.

16. Gómez, C. (2020). Enseñanza de valores mediante cuentos en Educación Infantil (Tesis de pregrado, Universidad de Valladolid). Repositorio Institucional. https://uvadoc.uva.es/handle/10324/40528

17. González, P. (2020). Las etapas del desarrollo emocional del niño. $\mathrm{https}$ ://eresmama.com/las-etapas-del-desarrollo-emocional-del-nino/.

18. Hernández, R., \& Mendoza, C. P. (2018). Metodología de la Investigación: las rutas cuantitativa, cualitativa y mixta. Mc Graw Hill.

19. Jiménez-Rodríguez, D. \& Osorio, G. (2016). Impacto de las habilidades sociales sobre el enojo: breve revisión teórica. Revista Electrónica de Psicología Iztacala, 19(2), 844-857.

20. López, P. (2014). Un caso de comportamiento disruptivo infantil: Tratamiento conductual en el ámbito familiar. Revista de Psicología Clínica con Niños y Adolescentes, 1(2), 117-123. https://www.revistapcna.com/sites/default/files/2-rpcna_vol.2_0.pdf.

21. Martínez, M. (2016). Estrategias de regulación emocional en niños escolarizados: diferencias según género y edad [Tesis de licenciatura, Universidad Nacional de Mar Plata] Facultad de psicología. Reposotorio institucional. http://rpsico.mdp.edu.ar

22. Marion, M. (1997). Guiding young children's understanding and management of anger. Young Children, 52(7), 62-67. https://eric.ed.gov/?id=EJ554448.

23. Moreno, A. (2017). Los cuentos como recurso para trabajar la ira en educación infantil [Tesis de pregrado, Universidad de Cantabria]. 
Repositorio

institucional.

https://repositorio.unican.es/xmlui/handle/10902/11790

24. Mytton, J., Diguiseppi, C., Gough, D., Taylor, R., \& Logan, S. (2002). School-based violence prevention programs: Systematic review of secondary prevention trials. Archives of Pediatrics and Adolescent Medicine, 156(8), 752-762. https://jamanetwork.com/journals/jamapediatrics/fullarticle/203682.

25. Oolup, C., Brown, J., Nowicki, E., \& Aziz, D. (2016). The Emotional Experience and Expression of Anger: Children's Perspectives. Child and Adolescent Social Work Journal, 33, 279-292. https://doi.org/10.1007/s10560-015-0423-3

26. Pérez, M. A., Redondo, M. M., \& León, L. (2008). Aproximaciones a la emoción de ira: de la conceptualización a la intervención psicológica. Revista Electrónica de Motivación y Emoción, 11(28), 119. http://reme.uji.es/articulos/numero28/article6/article6.pdf

27. Pérez, N. (2017). Lectura de álbumes infantiles para el control de la ira. [Tesis de posgrado, Universidad Pública de Navarra]. Repositorio institucional. https://hdl.handle.net/2454/25001

28. Piaget, J. (2017). Desarrollo Cognoscitivo. Enfoque piagetiano: el niño de las operaciones concretas. En D. Papalia \& G. Martorrell (Eds.). Desarrollo humano. McGraw-Hill Education.

29. Ramírez-Luna, J. O. (2018). Evaluación de la expresión del enojo y factores de riesgo en estudiantes de secundarias técnicas [Tesis de pregrado, Universidad Autónoma del Estado de Hidalgo]. Repositorio Institucional.

http://dgsa.uaeh.edu.mx:8080/bibliotecadigital/handle/231104/2191

30. Sánchez-Aragón, R. \& Díaz-Loving, R. (2009). El proceso de regulación emocional de la frustración y el enojo. Revista Mexicana de Investigación en Psicología, 1(1), 54-69.

31. Spielberger, C. D. (1999). Manual for the State-Trait Anger Expression Inventory-Revised. Psychological Assessment Resources.

32. Steinberg, N. (2018). El enojo en los niños. Doctora Nancy Steinberg. https://midoctoranancy.com/el-enojo-en-los-ninos/

33. Vicario, A. (2019). Literatura infantil y emoción: los cuentos infantiles de Trace Moroney como propuesta didáctica para el aula de primero de Educación Primaria. [Tesis de pregrado, Universidad de Cantabria] Repositorio institucional http://hdl.handle.net/10902/17705 